\title{
Modified Arytenoid Adduction Operation for the Treatment of Unilateral Vocal Fold Paralysis
}

\author{
Chao Liu a,b,c Yuanzheng Qiü,b,c Xin Zhanga,b,c Yong Liüa,b,c Guo Lia, b, c \\ Donghai Huang a, b, c \\ aDepartment of Otolaryngology Head and Neck Surgery, Xiangya Hospital, Central South University, Changsha, \\ China; ${ }^{b}$ Otolaryngology Major Disease Research Key Laboratory of Hunan Province, Changsha, China; ${ }^{\mathrm{c} C l i n i c a l}$ \\ Research Center for Pharyngolaryngeal Diseases and Voice Disorders in Hunan Province, Changsha, China
}

\section{Keywords}

Vocal cords paralysis · Arytenoid adduction · Voice quality

\begin{abstract}
Introduction: Unilateral vocal fold paralysis (UVFP) was a relative common glottic insufficiency disease; however, a completely satisfactory treatment of UVFP was elusive. This study was aimed to evaluate the surgical efficacy of modified arytenoid adduction with fenestration of the thyroid cartilage in the management of patients with UVFP, including voice and aspiration outcomes, and to summarize the postoperative complications. Methods: A retrospective analysis was performed on a total of 21 patients who underwent modified arytenoid adduction operation with fenestration of the thyroid cartilage for UVFP from July 2012 to June 2017. The scores of Grade, Roughness, Breathiness, Asthenia, Strain scale (GRBAS), voice self-satisfaction, dynamic laryngoscopy and the voice acoustic data (fundamental frequency [F0], fundamental frequency perturbation [jitter], loudness, amplitude perturbation [shimmer], and maximal phonatory time [MPT], etc.) were statistically analyzed preoperatively and 3-6 months postoperatively. The occurrence of postoperative complications was also summarized. Results: The voice subjective perception of 21 patients was significantly
\end{abstract}

improved after operation. The rate of voice self-satisfaction was $90.5 \%$. The mean values of voice acoustics parameters were significantly improved. The MPT was significantly longer $(p<0.001)$, and the ratings of postoperative aspiration were significantly decreased compared with the preoperation. Among the 21 patients, 15 cases had sense of laryngeal obstruction, 8 cases had of $1-2^{\circ}$ laryngemphraxis (recovered after 10-15 days). There were 2 cases of laryngeal stridor, 1 case of incision infection, 1 case of pharyngeal fistula, and 1 case of falsetto (corrected by voice training). No patient had laryngeal hematoma, neck hematoma, or laryngospasm. Conclusion: The modified arytenoid adduction operation with fenestration of the thyroid cartilage can significantly improve the vocal function of patients with UVFP and effectively reduce the aspiration, with fewer postoperative complications, less trauma, and more convenient advantages.

$$
\begin{aligned}
& \text { (c) } 2021 \text { The Author(s). } \\
& \text { Published by S. Karger AG, Basel }
\end{aligned}
$$

\section{Introduction}

Unilateral vocal fold paralysis (UVFP) is one of the most common glottic insufficiency diseases with the differences in the position and the height between the 2 vocal cords [1]. The most common reason for UVFP in-
(C) 2021 The Author(s).

Published by S. Karger AG, Basel

This is an Open Access article licensed under the Creative Commons Attribution-NonCommercial-4.0 International License (CC BY-NC) (http://www.karger.com/Services/OpenAccessLicense), applicable to the online version of the article only. Usage and distribution for commercial purposes requires written permission.
Correspondence to:

Donghai Huang, huangdonghai@csu.edu.cn 
cludes the recurrent laryngeal nerve injuries either as complications of neck and thoracic surgeries or from a neoplasm [2, 3]. These patients usually present with hoarseness, low voice, aspiration, speech difficulties, cough powerlessness, and deglutition disorder, which severely impair the quality of life [4]. Therefore, functional recovery of the paralyzed vocal fold is important for patients to lead better and fulfilling their lives.

The purpose of treating UVFP is to achieve optimum vocal cords closure for voice production [5]. Over the past few decades, the therapeutic strategies have advanced to multiple voice therapies and phonosurgical techniques, which include injection laryngoplasty, medialization thyroplasty, and arytenoid adduction [6]. However, none of these operations demonstrate to be definitely superior over the others [6]. Injection laryngoplasty treatment for UVFP often do not guarantee long-term effectiveness and widespread acceptance, and the traditional Type I thyroplasty cannot effectively correct differences in the levels of the 2 vocal folds [6-8]. Since first described by Isshiki et al. [9] in 1978, arytenoid adduction resulted in better correcting a large posterior glottal gap and vertical differences of the 2 vocal folds [10]. As the 3-dimensional movement of arytenoid was relatively complicated in phonation, the traditional Isshiki arytenoid adduction operation may generate unsatisfactory voice quality outcomes in some patients $[6,8]$. Meanwhile, compared with medialization thyroplasty, the disadvantages of increase in overall complications and operating time also restricted the widespread of arytenoid adduction operation [11]. Therefore, in this study, we modified the arytenoid adduction operation with fenestration of the thyroid cartilage to treat the patients with UVFP, which achieved satisfactory results with improvement of voice and aspiration and fewer postoperative complications.

\section{Materials and Methods}

\section{Patient Characteristics}

The subjects of our study included 21 patients with UVFP who had undergone the modified arytenoid adduction operation with fenestration of the thyroid cartilage from July 2012 to June 2017 (16 males and 5 females; age range 22-69 years with the median age of 50 years; 15 left and 6 right vocal fold paralysis). The reasons for vocal cord paralysis were as follows: 6 cases of injured recurrent laryngeal nerve by thyroid surgery, 4 cases of thyroid cancer invaded the recurrent laryngeal nerve, 2 patients of surgery for jugular foramen tumor, 1 patient of surgery for lung cancer, 1 patient of surgery for thymoma, and 7 cases of idiopathic neuritis. The duration of all these diseases lasted for 6 months to 7 years. The laryngoscopy examination showed all ipsilateral vocal cords were fixed to the paramedian or intermediate position with no significant atrophy. When patients pronounced/i:/, the vocal folds closed with obvious gap, and the affected vocal fold was arched with a certain height difference between the left and right vocal cords. In this study, all these 21 patients met the indications for modified arytenoid adduction operation, in which irreversible causes (disconnection of vagus or recurrent laryngeal nerve or tumor-infiltrating nerve in the surgery) or idiopathic neuritis were confirmed, and the hoarseness, choking cough, and other symptoms cannot be improved or compensated by systematic voice training for at least 6 months. The contraindications included the history was $<6$ months and the injuries of vagus and recurrent laryngeal nerve might be recovered, and those patients with radiotherapy in larynx, or previous surgery in the arytenoid cartilage, or destruction and trauma in the thyroid cartilage.

\section{Operation Procedure}

The arytenoid adduction was operated as described by Isshiki et al. [9] and Woodson et al. [12], with some modifications. Three patients were performed under local anesthesia with enhanced anesthesia, and the other 18 patients were given tracheal intubation general anesthesia. The operation procedures were as follows: (1) exposing the ipsilateral thyroid cartilage: a transverse incision of 5-6 cm long was created from $0.5 \mathrm{~cm}$ beside the median line of the thyroid cartilage in the intact side to the margin in the affected side (Fig. 1a), and for the patients with thyroid surgery, the incision was created along the original 1 . Then cut the skin, subcutaneous tissues and platysma, separate the platysma myocutaneous flap up to the plane of hyoid, down to the plane of the annular cartilage. Cut along the linea alba cervicalis, expose the ipsilateral thyroid cartilage (Fig. 1b), separate and protect the external branch of superior laryngeal nerve; (2) exposing the muscular process of arytenoid cartilage: $U$-shaped incision was created for the adventitia of thyroid cartilage with the pedicle in the median line. The arytenoid cartilage was located in the middle and lower $1 / 3$ level of the thyroid cartilage, anterior $2 / 3$ and posterior $1 / 3$ of the laminae of thyroid cartilage, which was positioned to penetrate a $1.0 \times 1.5 \mathrm{~cm}$ size of the cartilage window (Fig. 1c). Through the open window, cut the endometrium of thyroid cartilage and palpate the arytenoid cartilage, separate and expose the muscular process of arytenoid cartilage from the paralaryngeal space; (3) threading and fixation: following the confirmation of the muscular process, cut and expose the capsule of cricoarytenoid joint, and then loosen the arytenoid cartilage with stripper. Two 4-0 prolene sutures were placed through the muscular process. One of the sutures was parallel to the middle and lower $1 / 3$ level of the thyroid cartilage, whose end were passed through the holes drilled by a sharp stitch in the $0.5 \mathrm{~cm}$ beside the median line of the ipsilateral thyroid cartilage. The distance between these 2 stitches was about $0.5 \mathrm{~cm}$. Adjust the degree of tightness and adduction and place a self-shaped lamina of thyroid cartilage or silicone pad as a bolster between these 2 stitches. The other suture was attached to the posterior cricoid ligament to correct the plane of vocal cords (Fig. 1d). Recover the fenestrated cartilage and suture the adventitia of thyroid cartilage and then the linea alba cervicalis, platysma, subcutaneous, and skin were sutured layer by layer. The schematic diagram of the operation was shown in online suppl. Fig. 1; for all online suppl. material, see www.karger.com/doi/10.1159/000517561. 

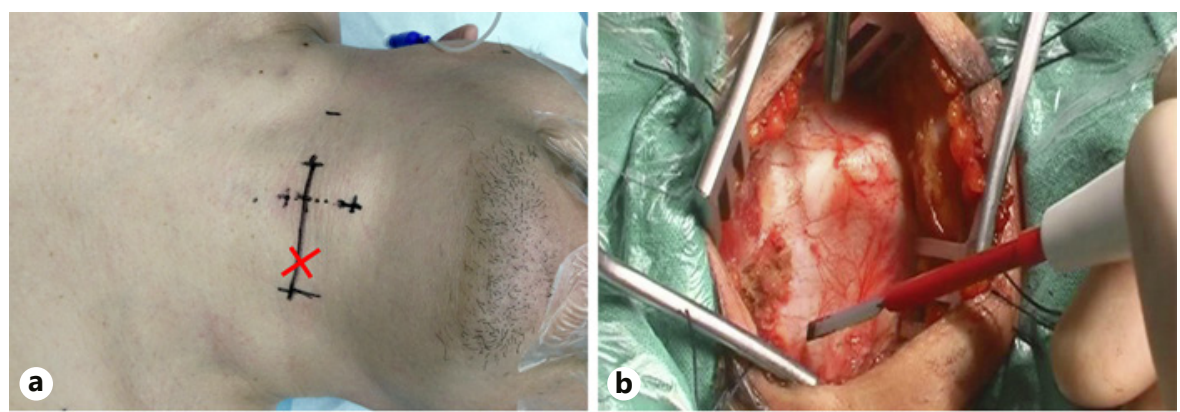

Fig. 1. Modified arytenoid adduction operation procedure for a representative case with UVFP. a Incision and the surface projection of arytenoid was shown. b Exposing the ipsilateral thyroid cartilage. c A window was created in the thyroid cartilage. d The position of sutures was shown. Black arrow: the fixation point of the laminae of thyroid cartilage; white arrow: the fixation point of the posterior cricoid ligament. UVFP, unilateral vocal fold paralysis.
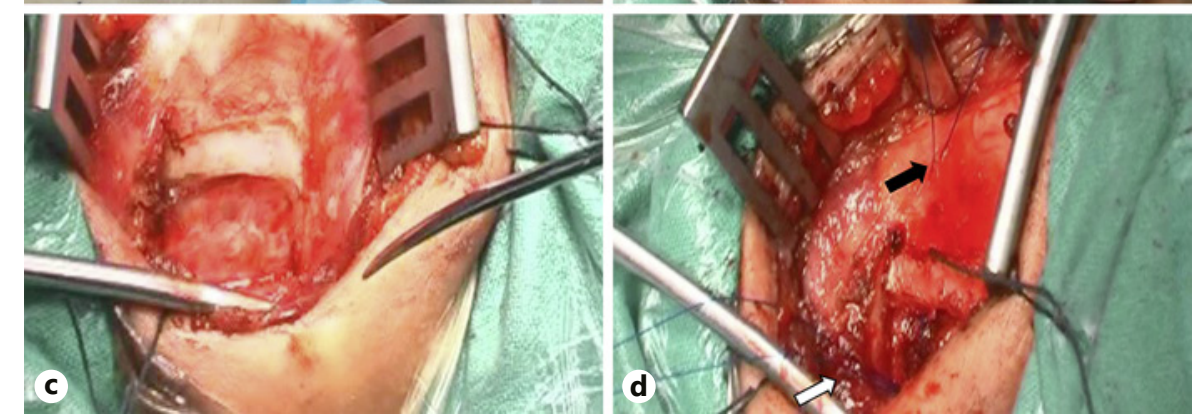

\section{Subjective Auditory Perception Assessment}

This assessment included the doctor's voice auditory perception assessment and the patient's voice self-satisfaction assessment. The laryngologist's voice auditory perception was evaluated by recording the patient's voice samples in a designated examination room with an ambient noise $<45 \mathrm{~dB}$ (A, and 2 experienced laryngologists blinded to the patient's clinical status scored independently as auditory judges. The GRBAS system proposed by Hirano [13] was adopted to evaluate the vocal function, which included 5 parameters of the scale of grade $(G)$, roughness $(R)$, breathiness (B), asthenia (A), and strain(S). A 4-point grading system including Grade 0 (normal), Grade 1 (mild), Grade 2 (moderate), and Grade 3 (severe) was used to quantify the voice auditory perception assessment.

Patients' self-satisfaction assessment was conducted independently by the patients themselves. The level of satisfaction was measured using 10-point scoring, in which 9-10 points represented very satisfied, $7-8$ points represented satisfaction, and 6 points and below represented not satisfied.

\section{Subjective Assessment of Aspiration}

Cummings [14] 4-point scale of aspiration with the score 0 (no indication), 1 (mild), 2 (moderate), and 3 (severe) was used to assess the severity of aspiration, in which level 1 indicated the subjective feeling of patients with occasional irritating cough but not needed to change the diet, level 2 referred to the severity of irritating cough with dietary modification, and level 3 (severe) meant the patients must require nasogastric tube nutrition.

\section{Dynamic Laryngoscopy and Voice Assessment}

This procedure was conducted by Germany XION dynamic laryngoscope. The most obvious adduction of vocal cord was assessed by taking the picture of the plane of the anterior and middle $1 / 3$ of the intact vocal cords, which was used as a unified measurement standard to measure the width of the glottic gap and com- pared between preoperatively and postoperatively. In brief, let the patient pronounce/i:/vowel comfortably, naturally, smoothly, and continuously, and then collected acoustic signals by dynamic laryngoscope system, and analyzed acoustic parameters including the fundamental frequency (F0), loudness, frequency perturbation (jitter, normal $<0.50 \%$ ), amplitude perturbation (shimmer, normal $<3 \%)$. For measurement of maximal phonatory time, let the patients pronounce/a:/vowel continuously as long as possible with a suitable tone and loudness after deep breathing, repeated the test 3 times and selected the strongest and the most stable signal for analysis (normal: male $>20 \mathrm{~s}$, female $>15 \mathrm{~s}$ ).

\section{Statistical Analysis}

All data were presented as mean \pm SD or grade data. Differences between 2 groups were compared using Student's $t$ test and Wilcoxon-rank sum test. The criterion for statistical significance was $p<0.05$. SPSS19.0 was used for statistical analysis.

\section{Results}

All these 21 patients with UVFP had undergone the modified arytenoid adduction operation with fenestration of the thyroid cartilage. The representative preoperative and postoperative electronic laryngoscopy examination was shown in online suppl. Video 1 and 2, and representative preoperative, and postoperative voice was shown in online suppl. Video 3 and 4.

\section{Subjective Auditory Perception Assessment}

Compared with the preoperation, the postoperative subjective auditory perception assessment results showed 
that the scores of GRBAS parameters were significantly decreased (Wilcoxon-rank sum test, $p<0.001$ ) (Table 1). For the patients' self-satisfaction assessment, 19 cases were very satisfied or satisfied, and the satisfaction rate was $90.5 \%(19 / 21)$.

\section{Aspiration Index Analysis}

After operation, there were 6 cases with mild aspiration, 3 cases with moderate aspiration, and the remaining 12 cases were without obvious aspiration. All patients with aspiration were recovered in 3 months after surgery, and the aspiration index was significantly decreased compared with preoperative (Wilcoxon-rank sum test, $Z=$ -2.876 ; $p=0.006$ ).

\section{Voice Acoustic Parameters Detection}

The results of voice acoustic parameters were summarized in Table 2, which demonstrated that compared with preoperative values, the voice acoustic parameters such as F0, basic frequency perturbation and amplitude perturbation were decreased significantly in 3 months after surgery and the loudness and maximal phonatory time were increased significantly (paired $t$ test, all $p<0.001$ ), which indicated that the modified arytenoid adduction operation could effectively improve the quality of voice in the patients with UVFP.

\section{Postoperative Complications}

The postoperative complications in all 21 patients including (1) laryngeal obstruction in 15 cases, and the incidence was $15 / 21=71.4 \%$. The symptoms usually disappeared from 10 days to 3 months after operation (2) $1-2^{\circ}$ laryngemphraxis in 8 cases, and the incidence was $8 / 21=$ $38.1 \%$. All cases improved in 10-15 days by anti-inflammatory and anti-edema treatment; (3) 2 cases of laryngeal stridor, and the incidence was $2 / 21=9.5 \%$, which occurred in $48 \mathrm{~h}-72 \mathrm{~h}$ after operation and improved in 7-10 days; (4) incision infection in 1 case, and the inci- dence was $1 / 24=4.8 \%$; (5) 1 case of pharyngeal fistula, and the incidence was $1 / 21=4.8 \%$; (6) 1 patient with falsetto. The male patient complained of 2 tones, a low male voice and a high pitched female voice, which was corrected by later pronunciation training; (7) laryngeal hematoma or neck hematoma in 0 cases; and (8) laryngospasm in 0 cases

\section{Discussion}

In UVFP patients, not only the paralyzed side is deviated significantly, there is also a significant height difference between the 2 vocal cords [1]. The surgical treatment is aimed to alleviate patients' hoarseness and prevent aspiration. Isshiki's classic arytenoid adduction surgery could not simulate the coordination movement between the adductors of the vocal folds (lateral cricoarytenoid muscle, arytenoid muscle, and thyroarytenoid muscle) and the abductor of the vocal folds (posterior cricoarytenoid muscle) [6]. So it often failed to close the posterior glottic gap effectively, which needed to be modified to better restore the original position of the vocal cords.

Woodson's arytenoid cartilage adduction operation can simulate the movement of the lateral cricoarytenoid muscle and posterior cricoarytenoid muscle during pro-

Table 1. GRBAS assessment of 21 patients between preoperatively and postoperatively

\begin{tabular}{lll}
\hline Parameter & $Z$ & $p$ value \\
\hline Grade (G) & -2.096 & $<0.001$ \\
Roughness (R) & -2.159 & $<0.001$ \\
Breathiness (B) & -2.279 & $<0.001$ \\
Asthenia (A) & -2.256 & $<0.001$ \\
Strain (S) & -2.109 & $<0.001$
\end{tabular}

Table 2. The comparison in voice acoustic parameters of 21 patients preoperatively and postoperatively

\begin{tabular}{lccccr}
\hline & F0, Hz & Loudness & Jitter, \% & Shimmer, \% & \multicolumn{1}{c}{ MPT, s } \\
\hline Preoperative & $231.12 \pm 74.39$ & $68.33 \pm 13.67$ & $1.74 \pm 1.23$ & $5.22 \pm 3.30$ & $6.11 \pm 2.17$ \\
3 months after surgery & $205.07 \pm 56.35$ & $76.89 \pm 9.11$ & $0.37 \pm 0.29$ & $1.72 \pm 1.13$ & $12.27 \pm 3.92$ \\
$t$ value & 5.18 & 4.899 & 5.211 & 4.922 & -12.16 \\
$p$ value & $<0.001$ & $<0.001$ & $<0.001$ & $<0.001$ & $<0.001$ \\
\hline
\end{tabular}

MPT, maximal phonatory time. 
nation and make the vocal folds in the physiological position to better close the glottic gap and correct the height difference of vocal cord $[12,15]$. In these cases, compared with the type I thyroplasty, Woodson's arytenoid cartilage adduction may not only effectively close the posterior glottic gap and restore normal vocal cord position, but the paralysis vocal cord can be also fixed to the intermediate position, thus improving the shape of pyriform sinus, reducing saliva retention, and improving swallow function and voice quality. Our study modified the Woodson's arytenoid cartilage adduction, in which the second suture was attached to the posterior cricoid ligament behind the posterior cricothyroid joint, rather than to the cornu inferius of the thyroid cartilage in Woodson's method. On the one hand, the modified arytenoid cartilage adduction can simulate the movement of the posterior cricoarytenoid muscle more effectively owing to the second suture towing in the same direction as this muscle. On the other hand, the suture in Woodson's method was attached to the cornu inferius of the thyroid cartilage, which was easy to loose or shift, and in our modified method, the suture was more accurate and tight, which could better restore the original position of vocal cords.

The perception assessment and voice acoustics analysis showed that the symptom of hoarseness in all patients were improved significantly, and the ability of glottis closure and phonation efficiency were also improved, which indicated that the modified arytenoid adduction treatment of UVFP can better improve the patient's voice function. Heitmiller et al. [16] found that the aspiration was occurred in $38 \%$ of UVFP patients. Compared with preoperatively, this group of patients' aspiration index was significantly improved, indicating that modified arytenoid adduction operation can reduce aspiration, restore strong cough function, thus improving the function of swallow and lung clearance.

The traditional medialization framework surgery of vocal folds required dissection of the inferior constrictor muscle of the pharynx, separating the mucosa of pyriform sinus, dragging the laminae of thyroid cartilage up to expose the laryngeal space, and then finding the arytenoid cartilage and its muscular process [6]. Dragging the thyroid cartilage flap was essential in this method; however, the thyroid cartilage flap was relatively wide and big in many patients, so it was a difficult and laborious job to drag and expose. In our method, we incised a small window on the thyroid cartilage directly to reach the paralaryngeal space, which facilitated to locate and expose the arytenoid cartilage and its muscular process simply and conveniently. Meanwhile, as the window was very small, it will not damage the framework of thyroid cartilage and did not affect its stability. This procedure was also validated as a fenestration approach in combined with type I thyroplasty by Tokashiki et al. $[17,18]$.

Compared with the thyroplasty, arytenoid adduction has not been widely carried out at present, probably due to technical challenge or the possibility of increased complications $[11,19]$. In all 21 cases in this study, no cases were found with severe laryngeal obstruction and hematoma. Postoperative antibiotics and steroid hormones could also reduce the incidence of infection and laryngeal edema, and negative pressure drainage in the neck could also prevent the occurrence of hematoma. Tracheotomy was an option for severe laryngeal edema and obstruction. The results in this study showed that in UVFP patients with obvious gap in glottic closure and significant height difference between the vocal cords, the modified arytenoid adduction operation can eliminate the wide posterior glottic gap, restore the original position of vocal cords, significantly improve the phonation function and prevent the aspiration. Therefore, the modified arytenoid adduction operation with fenestration of the thyroid cartilage is a safe and effective way for UVFP, yet the long-term effects still need to be further observed.

\section{Conclusion}

The modified arytenoid adduction operation with fenestration of the thyroid cartilage can significantly improve the voice and reduce the aspiration in the patients with UVFP, which may be a safe and effective treatment for UVFP.

\section{Statement of Ethics}

This study was conducted in accordance with the Declaration of Helsinki and approved by the Ethics Committee of Xiangya Hospital, Central South University (No. 201803649). Written informed consents were obtained from all the patients.

\section{Conflict of Interest Statement}

The authors declare that they have no conflict of interest.

\section{Funding Sources}

This study was supported by the Natural Science Foundation of Hunan Province (No. 2019JJ40481). 


\section{Author Contributions}

C.L. and D.H. were the major contributors in writing the manuscript. C.L., Y.Q., and X.Z. collected the patient data. C.L., Y.L., G.L., and D.H. analyzed the data and prepared the figures. C.L. and D.H. conceived the study and revised the final manuscript. All the authors read and approved the final manuscript.

\section{Data Availability Statementdata availability statement}

All data generated or analysed during this study are included in this article.

\section{References}

1 Atallah I, Manjunath MK, Castellanos PF. Transoral silastic medialization for unilateral vocal fold paralysis. Head Neck. 2019 Sep; 41(9):2947-51.

2 Matievics V, Bach A, Sztano B, Bere Z, Tobias Z, Castellanos PF, et al. Functional outcomes of endoscopic arytenoid abduction lateropexy for unilateral vocal cord paralysis with dyspnea. Eur Arch Otorhinolaryngol. 2017 Oct; 274(10):3703-10.

3 Chen HC, Pei YC, Fang TJ. Risk factors for thyroid surgery-related unilateral vocal fold paralysis. Laryngoscope. 2019 Jan;129(1): 275-83.

4 Zhou D, Jafri M, Husain I. Identifying the prevalence of dysphagia among patients diagnosed with unilateral vocal fold immobility. Otolaryngol Head Neck Surg. 2019 Jun; 160(6):955-64.

5 Walton C, Carding P, Flanagan K. Perspectives on voice treatment for unilateral vocal fold paralysis. Curr Opin Otolaryngol Head Neck Surg. 2018 Jun;26(3):157-61.

6 Siu J, Tam S, Fung K. A comparison of outcomes in interventions for unilateral vocal fold paralysis: a systematic review. Laryngoscope. 2016 Jul;126(7):1616-24.
7 Su CY, Tsai SS, Chuang HC, Chiu JF. Functional significance of arytenoid adduction with the suture attaching to cricoid cartilage versus to thyroid cartilage for unilateral paralytic dysphonia. Laryngoscope. 2005 Oct; 115(10):1752-9.

8 Shi J, Chen S, Chen D, Wang W, Xia S, Zheng $\mathrm{H}$. Modified arytenoid adduction for cancerrelated unilateral vocal fold paralysis. J Laryngol Otol. 2011 Feb;125(2):173-80.

9 Isshiki N, Tanabe M, Sawada M. Arytenoid adduction for unilateral vocal cord paralysis. Arch Otolaryngol. 1978 Oct;104(10):555-8.

10 Hong KH, Jung KS. Arytenoid appearance and vertical level difference between the paralyzed and innervated vocal cords. Laryngoscope. $2001 \mathrm{Feb} ; 111(2): 227-32$.

11 Abraham MT, Gonen M, Kraus DH. Complications of type I thyroplasty and arytenoid adduction. Laryngoscope. 2001 Aug;111(8): 1322-9.

12 Woodson G. Cricopharyngeal myotomy and arytenoid adduction in the management of combined laryngeal and pharyngeal paralysis. Otolaryngol Head Neck Surg. 1997 Mar; 116(3):339-43.

13 Hirano M. Psyco-acoustic evaluation of voice: GRBAS scale for evaluating the hoarse voice. Clinical examination of Voice: Springer Verlag; 1981.
14 Cummings CW, Purcell LL, Flint PW. Hydroxylapatite laryngeal implants for medialization. Preliminary report. Ann Otol Rhinol Laryngol. 1993 Nov;102(11):843-51.

15 Woodson GE, Picerno R, Yeung D, Hengesteg A. Arytenoid adduction: controlling vertical position. Ann Otol Rhinol Laryngol. 2000 Apr;109(4):360-4.

16 Heitmiller RF, Tseng E, Jones B. Prevalence of aspiration and laryngeal penetration in patients with unilateral vocal fold motion impairment. Dysphagia. 2000 Fall;15(4):184-7.

17 Tokashiki R, Hiramatsu H, Tsukahara K, Kanebayashi H, Nakamura M, Motohashi R, et al. A "fenestration approach" for arytenoid adduction through the thyroid ala combined with type I thyroplasty. Laryngoscope. 2007 Oct;117(10):1882-7.

18 Tokashiki R, Hiramatsu H, Shinada E, Motohashi R, Nomoto M, Toyomura F, et al. Analysis of pitch range after arytenoid adduction by fenestration approach combined with type I thyroplasty for unilateral vocal fold paralysis. J Voice. 2012 Nov;26(6):792-6.

19 Slavit DH, Maragos NE. Arytenoid adduction and type I thyroplasty in the treatment of aphonia. J Voice. 1994 Mar;8(1):84-91. 